\title{
El mercado del trabajo sexual femenino en la ciudad de San José, Costa Rica
}

\author{
Rosberly Rojas Campos, Julián Monge-Nájera, Iris Amalia Ramírez Sánchez y Roxana Morales Bonilla \\ Universidad Estatal a Distancia, 474-2050 San Pedro Montes de Oca, San José, Costa Rica; rrojas@uned.ac.cr; julianmonge@gmail.com; \\ iramirez@uned.ac.cr; rmorales@uned.ac.cr
}

Recibido 3-VI-2009 Corregido 1-IV-2009 Aceptado 7-V-2009

\begin{abstract}
The female sex work market in San Jose, Costa Rica. Sex work is a commercial transaction and is part of the economy, but social practices hide it and it is seldom mentioned in economic reports. In 2008 we interviewed 78 female sex workers in San José, capitol city of Costa Rica. They belonged in three groups: women working in the area surrounding the markets, fare US\$3 to US\$8 per hour and aged 26 to 81 years, workers at two massage parlors in San Juan de Tibás (US\$18 to US\$72 per hour, ages 18 to 33 years) and "call-girls" (US\$100 an hour; 19 to 38 years). The interviews were based on a questionnaire; respondents were paid their time, and data were analyzed with the SPSS statistical program. Depending on the group, between half and a majority consider sex work an acceptable way for women to obtain resources for their families, and for men, a sexual relief that reduces sexual violence. Women have no boss and decide independently on time and place of work. They reject customers if there is no agreement on which specific services they will provide. Few have experienced violence or sexually transmitted diseases as a result of their sex work. Most have no husband or permanent companion. The preferred client is kind, mature and deals always with the same woman. Undesirable customers are aggressive, mean, dirty and drugged. Customers are mostly married and aged 35-45 years. The requested services are diverse, but are dominated by oral sex and the "missionary" position. The rate is defined by group membership (market area, parlor or "call girl") and not by age. Earnings vary widely but generally are much higher than those for jobs appropriate to their low educational level. There is little culture of saving, and women have full decision on how they spend their earnings, which they mainly use to raise their children and support other relatives. Because few women do sex work, this activity represents a small proportion of economy in the city of San Jose.
\end{abstract}

KEY WORDS

Sex work, economy, market, supply, demand, price, savings, prostitution.

\section{Trabajo sexual femenino: perspectiva económica}

La economía trata la asignación de recursos escasos a necesidades ilimitadas. Aunque la disciplina nació hace más de 200 años al amparo de la filosofía moral, la teoría se ha desarrollado, básicamente, en relación con las decisiones productivas que involucran los recursos materiales. No es sino hasta mediados del siglo pasado, cuando algunos economistas (e.g. Becker 1973, 1976, McKenzie y Tullock 1978) estudiaron decisiones como el matrimonio y la criminalidad. Una evidencia de la aceptación que han tenido estas incursiones novedosas, es el otorgamiento del Premio Nobel de Economía de 1992 a Gary Becker por su aplicación del principio racional de comportamiento optimizador a la familia y al crimen y castigo.
En el caso del trabajo sexual, un tema tabú en prácticamente todas las sociedades, algunos economistas han aplicado modelos microeconómicos a las conductas de las mujeres que ofrecen estos servicios, los hombres que los demandan y el mercado en que se dan estas transacciones, pero el trabajo es muy poco considerando el tamaño del mercado. No hay datos exactos en el mundo acerca del número de mujeres que hacen trabajo sexual y menos aún del valor agregado que esta actividad representa en la producción mundial.

Con base en estadísticas nacionales sobre salud y condiciones socioeconómicas, Vandepitte et al. (2006) utilizaron datos de ciudades y zonas para calcular que, según el lugar, entre el 0,1 y el 7,4\% de las mujeres adultas del mundo hacen trabajo sexual (Cuadro 1). 


\section{CUADRO 1}

Estimaciones de la proporción de mujeres que hacen trabajo sexual, como porcentaje de la población de mujeres adultas, en diferentes regiones del mundo

\begin{tabular}{lcc}
\hline \multicolumn{1}{c}{ Región } & \multicolumn{2}{c}{ Proporciones (\%) } \\
& Límite inferior & Límite superior \\
\hline Belice & 7,4 & 7,4 \\
Haití & 2,0 & 2,0 \\
República Dominicana & 1,8 & 1,8 \\
Venezuela & 1,5 & 1,5 \\
África Subsahariana & 0,7 & 4,3 \\
(Ciudades capitales) & & \\
Colombia & 0,7 & 0,7 \\
África Subsahariana & 0,4 & 4,3 \\
(Otras ciudades) & & \\
Europa del Este & 0,4 & 1,4 \\
Perú & 0,3 & 0,3 \\
Asia & 0,2 & 2,6 \\
América Latina & 0,2 & 7,4 \\
Bolivia & 0,2 & 0,2 \\
Ex-Federación Rusa & 0,1 & 1,5 \\
Europa occidental & 0,1 & 1,4 \\
\hline
\end{tabular}

FUENTE: Elaboración a partir de los datos presentados por Vandepitte et al. 2006

La importancia de este sector económico, al igual que el de otras actividades de la economía informal, ha sido reconocida por organismos internacionales como la Organisation for Economic Cooperation and Development (OECD 2002). La mayoría de los países aún no lo incluyen, pero sabemos que por ejemplo en Polonia el trabajo sexual ocupó 0,2\% del Producto Interno Bruto (PIB) en 1998 (OECD 2002).

Para el caso de Costa Rica no se disponen datos sobre el aporte al PIB, aunque la tasa en población urbana se estima en 2 trabajadoras por cada 1000 habitantes (Acuña et al. 1982).

\section{Teoría económica sobre trabajo sexual}

A principios de la década de 1990, el libro Sexo y $R a-$ zón presentó una teoría económica de la sexualidad, estratificando el mercado de los EEUU en: pública, referente a mujeres que esperan a sus clientes en las aceras; de clubes, donde los clientes acuden a un lugar, y personalizada, en el cual la trabajadora acude al lugar donde el cliente se hospeda o habita ("call-girls") (Posner 1992).

La mayoría de hombres prefieren la fidelidad en sus esposas y para ello están dispuestos a incurrir en gastos de manutención familiar asociado con el sexo reproductivo. Se define el trabajo sexual como el acto de brindar, desde el punto de vista del cliente, sexo no reproductivo a cambio de un pago (Edlund y Korn 2002). El trabajo sexual es una actividad económica intensiva en trabajo, principalmente femenina que no requiere alta calificación pero es muy bien pagada. La explicación de esta aparente contradicción está en el mercado de matrimonios: si una mujer se dedica al trabajo sexual, reduce sensiblemente sus posibilidades de ser esposa. Por tanto, hay un costo de oportunidad que explica la diferencia salarial (Edlund y Korn 2002).

La oferta de servicios sexuales está determinada por las opciones de trabajo y sus condiciones, los salarios alternativos, y el estigma social que implica el trabajo sexual (Della et al. 2005). En México, algunas trabajadoras sexuales recibieron $23 \%$ de pago adicional por sexo sin la protección del condón y ese porcentaje subió a $46 \%$ si la trabajadora era considerada muy atractiva (Gertler et al. 2005). En India, los determinantes de los precios son la duración del servicio, la localización, y la edad de la mujer, pero la satisfacción del cliente no afecta el precio (Della et al. 2005).

Hay aún menos estudios sobre el cliente. Las principales razones por la que éste busca servicios sexuales (Weitzer 2005) son:

- Obtener prácticas sexuales específicas, como el sexo oral (felación).

- Tener una mayor variedad en sus experiencias sexuales.

- Tener actividad sexual con una persona con cierta imagen (e.g., atractiva, vulgar) o atributos físicos (e.g. color de piel, transexualidad).

- Por el atractivo de lo "ilícito y riesgoso".

- Evitar relaciones emocionales y sus obligaciones.

- Dificultad para establecer relaciones convencionales.

El 76\% de hombres en dos ciudades de EEUU, arrestados por solicitar servicios sexuales, dijeron que no se casarían con una de estas trabajadoras (Weitzer 2005).

\section{Estudios sobre trabajo sexual en el ámbito costarricense}

En Costa Rica, hay estudios basados en historias de vida (Herrera 2000, Sisa 2006) y en la historia de esta actividad económica durante los últimos siglos (Fernández y Rodríguez 2005, Marín 2005), pero los únicos estudios relacionados con la economía son encuestas de características socioeconómicas de las trabajadoras de San José (Acuña et al. 1982, Ortiz et al. 1998)

En este artículo analizamos los factores económicos que, iniciando el siglo XXI, determinan el mercado de 
servicios sexuales en la ciudad de San José, incluyendo la historia personal de las trabajadores y algunas características y deseos de los clientes. Es el primer estudio de su tipo en Costa Rica.

\section{METODOLOGÍA}

Entrevistamos a tres grupos de trabajadoras del centro de la ciudad de San José, Costa Rica, en 2008.

\section{Grupo 1: Ambulantes}

Mujeres que trabajan en la zona aledaña al Mercado Borbón y Mercado Central, con tarifa entre US\$3 y US\$8 por hora y edades de 26 a 81 años. Su apariencia física y nivel educativo no alcanzan el nivel de los otros grupos.

\section{Grupo 2: De Sala}

Trabajadoras de dos salas de masajes de San Juan de Tibás. Sus tarifas oscilan de US\$18 a US\$72 por hora y sus edades de 18 a 33 años. Su apariencia física y nivel educativo son intermedios.

\section{Grupo 3: VIP}

Trabajadoras independientes que cobran US\$100 la hora, tienen entre 19 y 38 años de edad y atienden clientes de alto poder equisitivo, a menudo fijos. Su apariencia física y nivel educativo son los más altos de los tres grupos.

Las entrevistas se hicieron con base en un cuestionario, se pagó a las entrevistadas su tiempo según la misma tarifa que pagaría un cliente, y los datos se analizaron con el programa estadístico SPSS. Los porcentajes y relaciones que se presentan con respecto al total de respuestas válidas para cada pregunta, excluyendo los valores faltantes. Los detalles metodológicos se presentan en Ramírez et al. (2009).

\section{RESULTADOS}

\section{El trabajo sexual como actividad económica}

La mitad de las trabajadoras del grupo 1 visualizaron su trabajo como actividad productiva. En el grupo 2 predomina la percepción positiva de su labor como trabajo productivo, contrario al grupo 3 (Fig. 1).

El subgrupo que no considera el trabajo sexual como trabajo (Fig. 2) hizo comentarios como estos:

- "Es un trabajo sucio, en donde la plata es sucia y no rinde para nada"

. "Una mujer no debería venderse"

. "El dinero no se gana por bien"

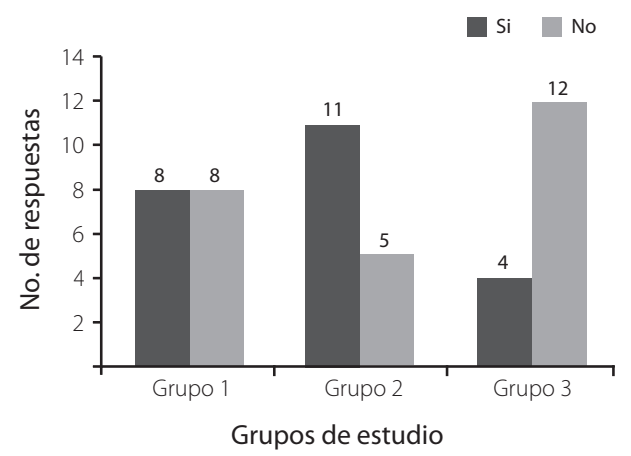

FIG. 1. Mujeres entrevistadas por grupo, según consideren o no su actividad como trabajo productivo.

- Hay que "aguantar cosas denigrantes"

Otra razón, también frecuente, es el riesgo propio de la actividad, que puede ser por contraer enfermedades, porque el cliente sea agresivo, o incluso por la posibilidad de enamorarse de un cliente. El rechazo de la sociedad también fue mencionado.

Las trabajadoras del grupo 3 mencionaron el no estar aseguradas y el nivel de exigencia física.

La mayoría dijo que su trabajo es necesario para la sociedad (Cuadro 2).

Aunque una tercera parte de las entrevistadas dijo que la actividad no es necesaria para la sociedad, la mayoría no dio razones; dos dijeron que ofrecen sus servicios solo por necesidad, una que la persona se "devalúa" al prestar estos servicios y una que los hombres se "limitarían a sus esposas" si ellas no vendieran sus servicios.

En cuanto a la justificación de su actividad como socialmente necesaria (Cuadro 3), las razones varían entre los grupos estudiados. En el grupo 1, el principal argumento se basa en que al satisfacer los hombres sus deseos sexuales con las mujeres que hacen trabajo sexual, se evitan los abusos sexuales y las violaciones a mujeres y niñas/os. Para el grupo 2, el criterio principal es que la actividad es una buena opción para obtener ingresos y permite a los hombres escapar de la rutina. En el grupo 3, que hay una demanda importante de sus servicios, ya que los hombres, por naturaleza, requieren variedad de mujeres.

\section{La oferta de servicios sexuales}

Dado que los servicios sexuales son de trabajo intensivo, se presentan aquí la jornada laboral, las relaciones de dependencia, la libre elección y los costos de oportunidad (vida en pareja y riesgos). 


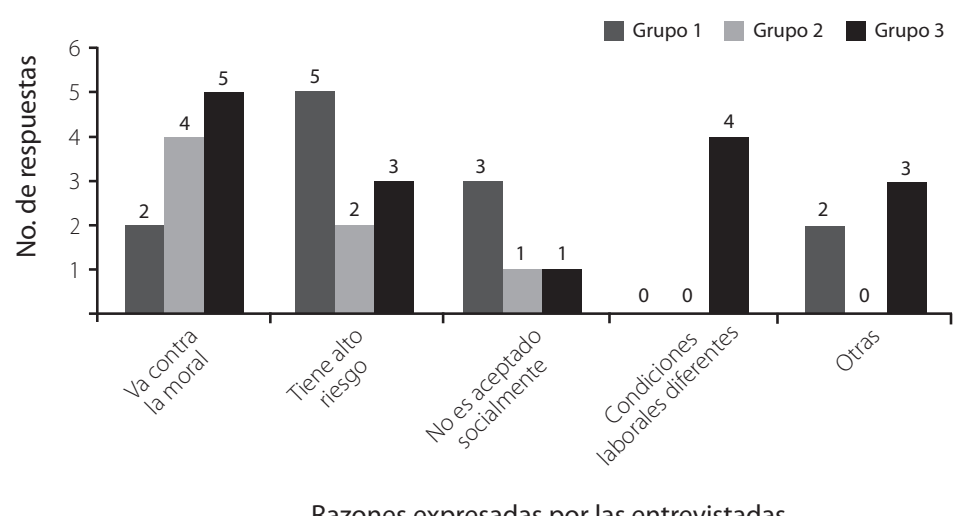

Razones expresadas por las entrevistadas

FIG. 2. Mujeres entrevistadas por grupo, según las razones expresadas para no considerar su actividad como productiva.

CUADRO 2

Mujeres entrevistadas por grupo, según su opinión sobre la necesidad social de su actividad (en porcentajes)

\begin{tabular}{lcccc}
\hline \multicolumn{1}{c}{ Opinión } & Grupo 1 & Grupo 2 & Grupo 3 & Total \\
\hline $\begin{array}{l}\text { Si es necesaria } \\
\text { socialmente }\end{array}$ & 63,2 & 83,3 & 53,3 & 67,3 \\
$\begin{array}{l}\text { No es necesaria } \\
\text { socialmente }\end{array}$ & 36,8 & 16,7 & 46,7 & 32,7 \\
\begin{tabular}{l} 
Total \\
\hline
\end{tabular} & 100,0 & 100,0 & 100,0 & 100,0 \\
\hline
\end{tabular}

CUADRO 3

Razones para considerar su actividad como una necesidad social (en porcentajes)

\begin{tabular}{lcccc}
\hline \multicolumn{1}{c}{ Razones } & Grupo 1 & Grupo 2 & Grupo 3 & Total \\
\hline $\begin{array}{l}\text { Evita violaciones } \\
\text { y abusos de los } \\
\text { hombres a mujeres } \\
\text { y niños/as }\end{array}$ & 55,6 & 18,8 & 16,7 & 29,0 \\
$\begin{array}{l}\text { Opción de las } \\
\text { mujeres para } \\
\text { obtener ingresos }\end{array}$ & 33,3 & 31,3 & 33,3 & 32,3 \\
$\begin{array}{l}\text { Existe demanda } \\
\text { en el mercado }\end{array}$ & 11,1 & 25,0 & 50,0 & 25,8 \\
$\begin{array}{l}\text { Permite escapar de la } \\
\text { rutina a los hombres }\end{array}$ & - & 25,0 & - & 12,9 \\
\begin{tabular}{l} 
Total \\
\hline
\end{tabular} & 100,0 & 100,0 & 100,0 & 100,0 \\
\hline
\end{tabular}

\section{Jornada de trabajo}

Las oferentes de este servicio laboran en promedio 9 $1 / 2$ horas diarias (desviación estándar 2,8; mínimo 4 horas y máximo 15 horas). Promedio por grupo (1 al 3 respectivamente) son 8,10 y 11 horas.

No hay un horario fijado por un patrono. Un 64\% tiene un horario fijo, pero en todos los casos la jornada es determinada por la mujer, de acuerdo con sus condiciones personales y el tipo de población al que se dirigen sus servicios.

Prevalece el horario que combina las horas del día y de la tarde (Cuadro 4). Las mujeres del grupo 1 tienen preferencia por el horario diurno (40\%), según algunas manifiestan, debido al riesgo de la zona donde trabajan. Las del grupo 2 optan (80\%) por el diurno y vespertino, ya que la mayoría de ellas tratan de ocultar su actividad económica y manifiestan en sus familias que laboran en otras actividades tales como los centros de llamadas y restaurantes. En el grupo 2, si bien la mujer define su horario, este se debe adecuar a las horas en que el local que alquila esté abierto. Las del grupo 3 están más dispuestas a trabajar tanto de día como de noche (58,8\%), mayor flexibilidad que está considerada en la tarifa cobrada, ya que en muchos casos la demanda de sus servicios proviene de turistas extranjeros, quienes las contratan para la salida de la noche o incluso para pasar todo el fin de semana con ellas, a menudo en playas y otros sitios turísticos, a los que acuden con todos los gastos pagos además de su tarifa laboral. 


\section{CUADRO 4}

Mujeres entrevistadas por grupo, según los tipos de horario en que prestan sus servicios (en porcentajes)

\begin{tabular}{lcccc}
\hline \multicolumn{1}{c}{ Tipo de horario } & Grupo 1 & Grupo 2 & Grupo 3 & Total \\
\hline Diurno & 40,0 & 10,0 & - & 15,4 \\
Vespertino & 20,0 & - & - & 5,8 \\
Nocturno & 20,0 & - & 11,8 & 9,6 \\
Diurno/nocturno & 20,0 & 10,0 & 58,8 & 28,8 \\
Diurno/vespertino & - & 80,0 & 23,5 & 38,5 \\
Vespertino/nocturno & - & - & 5,9 & 1,9 \\
Total & 100,0 & 100,0 & 100,0 & 100,0 \\
\hline
\end{tabular}

Horario diurno: 7 de la mañana hasta las 6 de la tarde, vespertino de las 12 mediodía hasta las 8 de la noche y nocturno de 8 en adelante.

\section{Relaciones de dependencia laboral}

La mayoría de las entrevistadas no trabaja para una persona que actúa comojefe, lo hace independientemente. En el grupo 1 solo dos mujeres, de las 20 entrevistadas, expresaron trabajar en un lugar en el cual hay una administradora del sitio donde ofrecen sus servicios, con quien tienen buenas relaciones. En el grupo 2, subcontratan el espacio físico para ofrecer sus servicios, de manera que pagan por el uso de la casa. En el grupo 3, dos mujeres manifestaron trabajar para intermediarios, quienes son los que cobran por los servicios a los clientes y luego pagan a ellas una cantidad de dinero, otra trabaja en un "Night Club" y sus dueños actúan como jefes (incluso las amonestan pero incumplen sus obligaciones legales como patronos).

\section{Libre elección en la prestación de servicios}

Existe cierto grado de libre elección en la prestación del servicio, por ambas partes. Los clientes escogen a la mujer y la mujer puede rechazar ciertas solicitudes del cliente e incluso negarse a atender a un cliente. Esta libre elección supera el $80 \%$ en los grupos 2 y 3 y aunque en el grupo 1 es menor debido a la mayor necesidad de obtener ingresos, aun en ese grupo el $74 \%$ de las mujeres rechaza sistemáticamente aquellos servicios que no desea dar (Fig. 3).

La motivación de un pago adicional a la tarifa por brindar un tipo de servicio no deseado por la mujer que hace trabajo sexual es más importante en el grupo 1, donde un $22,2 \%$ estará dispuesta a brindarlo a cambio de dinero extra, sin embargo esta motivación se reduce sensiblemente en los grupos 2 y 3 ( $15,8 \%$ y $5,6 \%$ respectivamente).

\section{Costos de oportunidad en la prestación de servicios}

El que las mujeres se dediquen a la prestación de servicios sexuales puede implicar un costo de oportunidad para ellas en relación con la disminución de la posibilidad de tener una vida sentimental en pareja, especialmente de tipo matrimonial. Por ello, se presenta la información respecto a las relaciones de pareja que tienen las entrevistadas y sus opiniones sobre los riesgos laborales.

\section{Posibilidad de vida sentimental en pareja}

Un $68,5 \%$ dijeron no tener una relación de pareja actualmente (Fig. 4). De quienes la tienen, 16,7\% tiene novio o compañero (a veces conviven) y $14,8 \%$ dicen tener esposo. Según datos del Censo de Población de Costa Rica, del año 2000, un 48,5\% de las mujeres mayores

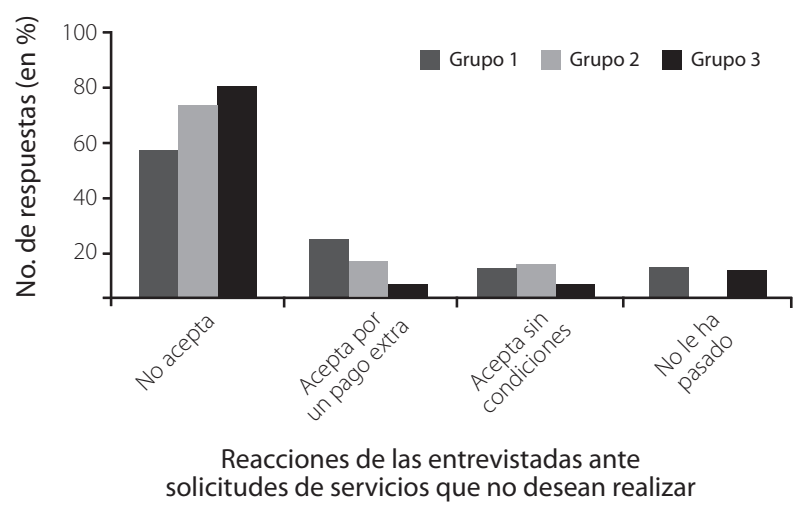

FIG. 3. Mujeres entrevistadas por grupo, según su respuesta ante la solicitud de un servicio que no desea realizar (en porcentajes). 
de 12 años, no tienen ninguna relación de pareja (INEC 2000), por ende, entre las trabajadoras sexuales es mucho mayor el porcentaje.

\section{El riesgo asumido en la prestación del servicio}

Las mujeres entrevistadas consideraban el que se experimentaban riesgos mucho mayores que en otros trabajos: contraer enfermedades, agresividad de sus clientes y riesgo emocional (posibilidad de enamorarse de un cliente).

En cuanto al riesgo de contraer enfermedades de transmisión sexual, son mínimas y el grupo que más lo ha padecido es el grupo 1; sin embargo, al igual que los otros dos grupos existe un amplio uso del condón y las visitas de control médico (Fig. 5)

La mayoría de las mujeres nunca ha sufrido agresiones físicas o verbales de parte de los clientes en su trabajo.

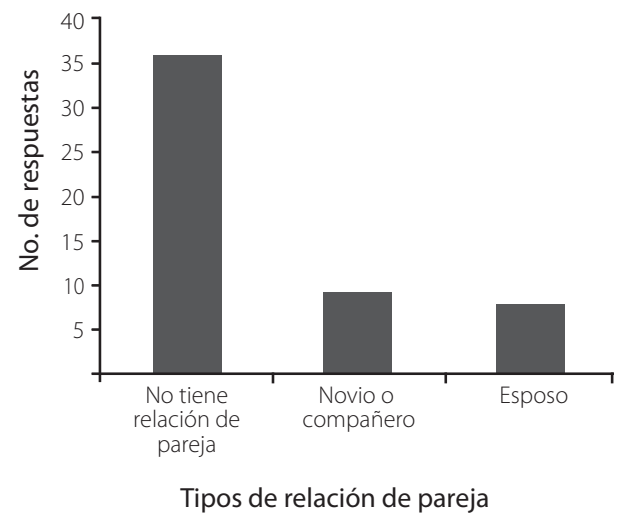

FIG. 4. Mujeres entrevistadas según hayan tenido relaciones de pareja.

Un 34,5\% han sufrido agresión física por lo menos una vez y un $43,6 \%$ agresión verbal por lo menos una vez en todos sus años de trabajo, aunque todas afirman que estas agresiones son la excepción, no la regla. Como agresiones físicas se incluyeron golpes, pellizcos, empujones y otros. Como agresiones verbales se especificaron los insultos, las amenazas, las acusaciones y las risas burlonas. Han sufrido ambos tipos de agresión al menos una vez en todos sus años de trabajo un 10,9; un 9,1 y un 7,3\% (grupos 1 al 3, respectivamente; Cuadro 5 ).

El riesgo de enamorarse de los clientes es real: en el grupo 1 le ha ocurrido a la mitad de las mujeres, en el 2 al $30 \%$ y en el 3 al $33,3 \%$.

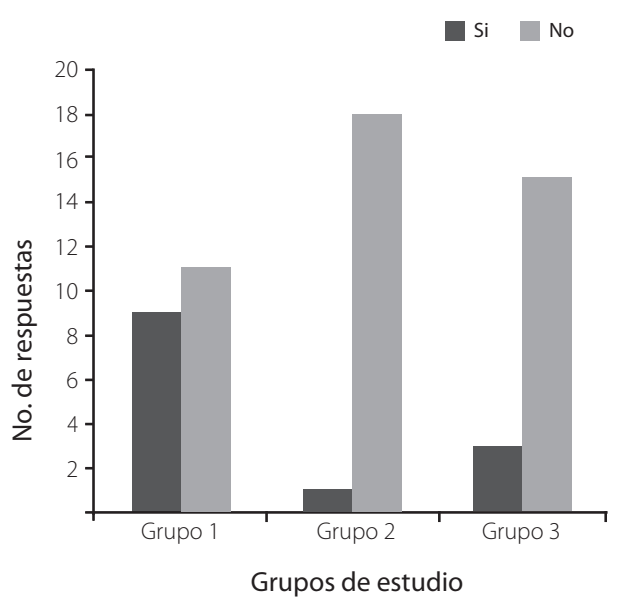

FIG. 5. Mujeres entrevistadas por grupo, según hayan sufrido enfermedades de transmisión sexual

\section{Los demandantes del servicio}

Los demandantes de los servicios sexuales, según las entrevistadas, son en general de sexo masculino. Para ellos, se presenta la valoración que hicieron las entrevistadas de los tipos de clientes, sus edades, el estrato socioeconómico, y su estado civil.

\section{Mejores y peores tipos de clientes}

Caracterizan al buen cliente el trato amable, la procedencia extranjera, el que sea cliente conocido y la edad madura. En el grupo 1, el mejor es aquel que les da un buen trato y le sigue el cliente "permanente" a quienes en las entrevistas se referían como amigos. En el caso del grupo 2, el trato recibido. En el grupo 3, el "gringo" (estadounidense o europeo) (Cuadro 6).

\section{CUADRO 5}

Mujeres entrevistadas por grupo, según hayan recibido agresión física y verbal (en porcentajes)

\begin{tabular}{|c|c|c|c|c|c|}
\hline \multirow{2}{*}{ Grupo } & & & \multicolumn{2}{|c|}{ Agresión verbal } & \multirow{2}{*}{ Total } \\
\hline & & & No & $\mathrm{Si}$ & \\
\hline \multirow{2}{*}{1} & \multirow{2}{*}{$\begin{array}{l}\text { Agresión } \\
\text { física }\end{array}$} & No & 14,5 & 7,3 & 21,8 \\
\hline & & $\mathrm{Si}$ & 1,8 & 10,9 & 12,7 \\
\hline \multirow{2}{*}{2} & \multirow{2}{*}{$\begin{array}{l}\text { Agresión } \\
\text { física }\end{array}$} & No & 20,0 & 5,5 & 25,5 \\
\hline & & $\mathrm{Si}$ & 1,8 & 9,1 & 10,9 \\
\hline \multirow{3}{*}{3} & \multirow{2}{*}{$\begin{array}{l}\text { Agresión } \\
\text { física }\end{array}$} & No & 16,4 & 3,6 & 20 \\
\hline & & $\mathrm{Si}$ & 1,8 & 7,3 & 9,1 \\
\hline & Total & & 56,3 & 43,7 & 100 \\
\hline
\end{tabular}


Los peores clientes son los agresores (grupo 2), seguidos de los que piden un servicio muy completo pero no desean pagar la tarifa y menos aún dar propinas, y los de apariencia desagradable por desaseo o drogadicción (grupos 1 y 3; Cuadro 7).

\section{Edad de los clientes}

Las entrevistadas de los tres grupos manifiestan que tienen clientes de todas las edades, sin embargo los datos muestran ligeras diferencias, pues en el grupo 1 el ámbito de edades va de 15 a 90 años, en el grupo 2 de 18 a 75 años y en el grupo 3 de 20 a 60 años.

\section{CUADRO 6}

Mejor tipo de cliente, según criterio de las entrevistadas (en porcentajes)

\begin{tabular}{lcccc}
\hline \multicolumn{1}{c}{ Mejores clientes } & Grupo 1 & Grupo 2 & Grupo 3 & Total \\
\hline $\begin{array}{l}\text { Quienes las tratan } \\
\text { bien }\end{array}$ & 45,0 & 80,0 & 27,8 & 51,7 \\
$\begin{array}{l}\text { Extranjeros que } \\
\text { pagan bien }\end{array}$ & 15,0 & 10,0 & 61,1 & 27,6 \\
$\begin{array}{l}\text { Clientes conocidos } \\
\text { Adultos mayores }\end{array}$ & 30,0 & - & 11,1 & 13,8 \\
Total & 10,0 & 10,0 & - & 6,9 \\
\hline
\end{tabular}

\section{Estado civil de los clientes}

Las mujeres entrevistadas opinan que los demandantes de sus servicios en su mayoría son casados, aunque atienden personas de todo estado civil (Cuadro 8).

\section{Nacionalidad de la clientela}

La mayoría de las entrevistadas tienen clientes nacionales y extranjeros, aunque en el grupo 2 se da una predominancia de clientela nacional (Cuadro 9).

\section{El servicio transado}

El servicio es individualizado. Consultamos sobre los servicios solicitados por el cliente más reciente y sobre los más frecuentemente solicitados en general.

\section{Servicios solicitados por el cliente más reciente}

Los tres servicios más frecuentemente solicitados son el coito tradicional (i.e. vaginal), el sexo oral y los masajes con caricias y besos (Cuadro 10).

En general, los servicios solicitados son muy variados (Cuadro 11).
CUADRO 7

Peor tipo de cliente, según criterio de las entrevistadas (en porcentajes)

\begin{tabular}{lcccc}
\hline \multicolumn{1}{c}{ Peores clientes } & Grupo 1 & Grupo 2 & Grupo 3 & Total \\
\hline Agresivos & 31,6 & 70,0 & 16,7 & 40,4 \\
$\begin{array}{l}\text { Exigen mucho y } \\
\text { pagan mal }\end{array}$ & 36,8 & - & 50,0 & 28,1 \\
Sucios y adictos & 10,5 & 20,0 & 22,2 & 17,5 \\
Otros & 15,8 & 10,0 & - & 8,8 \\
Ninguno & 5,3 & - & 11,1 & 5,3 \\
Total & 100,0 & 100,0 & 100,0 & 100,0 \\
\hline
\end{tabular}

\section{CUADRO 8}

Estado civil de los clientes, según criterio de las entrevistadas (en porcentajes)

\begin{tabular}{lcccc}
\hline $\begin{array}{c}\text { Estado civil } \\
\text { del cliente }\end{array}$ & Grupo 1 & Grupo 2 & Grupo 3 & Total \\
\hline Casados & 36,8 & 88,2 & 58,8 & 60,4 \\
De todos & 52,6 & 11,8 & 17,6 & 28,3 \\
Solteros & 10,5 & 0,0 & 11,8 & 7,5 \\
Divorciados & 0,0 & 0,0 & 11,8 & 3,8 \\
Total & 100,0 & 100,0 & 100,0 & 100,0 \\
\hline
\end{tabular}

CUADRO 9

Nacionalidad de los clientes, según criterio de las entrevistadas (en porcentajes)

\begin{tabular}{lcccc}
\hline $\begin{array}{l}\text { Nacionalidad } \\
\text { de los clientes }\end{array}$ & Grupo 1 & Grupo 2 & Grupo 3 & Total \\
\hline Costarricenses & 7,7 & 88,9 & 26,7 & 45,7 \\
Ambos & 92,3 & 11,1 & 40,0 & 43,5 \\
Extranjeros & - & - & 33,3 & 10,9 \\
Total & 100,0 & 100,0 & 100,0 & 100,0 \\
\hline
\end{tabular}

De los 12 servicios solicitados que no incluyeron el coito tradicional, las preferencias de los clientes se orientan hacia el sexo oral (8 de 12 casos), los masajes, caricias y besos ( 6 de 12) y las posiciones diferentes a la tradicional (4 de 12).

Predominan los servicios constituidos por una actividad. Le siguen los compuestos por dos $(30,4 \%)$ y tres actividades (26,1\%). Los servicios muy diversificados, de 4 o 5 actividades, ocupan un $11 \%$ de los casos (Cuadro 12). 
CUADRO 10

Tipo de servicio brindado al último cliente $(\mathrm{N}=46)$

\begin{tabular}{lc}
\hline \multicolumn{1}{c}{ Tipo de servicio } & Porcentaje \\
\hline Solamente coito tradicional & 28,3 \\
Coito tradicional y sexo oral & 6,5 \\
Coito tradicional, masajes, & 6,5 \\
caricias y besos, sexo oral & \\
Otros & 58,7 \\
Total & 100 \\
\hline
\end{tabular}

\section{El servicio más frecuentemente solicitado}

Los dos servicios más solicitados son el sexo oral y el coito tradicional, esto según la cantidad de menciones hechas por las entrevistadas sobre esas dos actividades, como solicitadas por los clientes.

Al igual que en el caso de los servicios demandados por el último cliente, 33,9\% de las mujeres dijeron que son muy solicitadas actividades de comunicación y relación como masajes, caricias y besos; la conversación y compartir una cena.

Para todos estos tipos de servicios, se incluye el uso del condón: 52 de las 58 mujeres entrevistadas mencionan que lo usan para protegerse de enfermedades de transmisión sexual (ETS). Únicamente seis (2,8\%) dijeron estar dispuestas a un pago extra por no usar el condón (Cuadro 13).

CUADRO 11

Actividades presentes dentro de los servicios $(\mathrm{N}=46)$

\begin{tabular}{lc}
\hline \multicolumn{1}{c}{ Actividad } & Porcentaje \\
\hline Coito tradicional & 73,9 \\
Sexo oral & 43,5 \\
Masajes, caricias, besos & 34,8 \\
Posiciones diferentes & 21,7 \\
Relación con dos mujeres & 8,7 \\
Masturbación & 6,5 \\
Conversación & 6,5 \\
Salir a cenar & 4,3 \\
Ver pornografía & 4,3 \\
Sexo anal & 2,2 \\
Juego de roles & 2,2 \\
Salir a bailar & 2,2 \\
\hline
\end{tabular}

CUADRO 12

Composición de los servicios $(\mathrm{N}=46)$

\begin{tabular}{|c|c|}
\hline Composición del servicio & Porcentaje \\
\hline Servicios compuestos por una actividad & 32,7 \\
\hline Coito tradicional & 28,3 \\
\hline Sexo oral & 2,2 \\
\hline Relación con dos mujeres & 2,2 \\
\hline Servicios compuestos por dos actividades & 30,4 \\
\hline Coito tradicional y sexo oral & 6,5 \\
\hline Coito tradicional y masajes & 4,3 \\
\hline Coito tradicional y relación con dos mujeres & 4,3 \\
\hline Posiciones diferentes y sexo oral & 4,3 \\
\hline Coito tradicional y cenar juntos & 2,2 \\
\hline Posiciones diferentes y masajes & 2,2 \\
\hline Masajes y sexo oral & 2,2 \\
\hline Masajes y masturbación & 2,2 \\
\hline Masajes y conversación & 2,2 \\
\hline Servicios compuestos por tres actividades & 26,1 \\
\hline $\begin{array}{l}\text { Coito tradicional, posiciones diferentes } \\
\text { y sexo oral }\end{array}$ & 4,3 \\
\hline Coito tradicional, masajes, sexo oral & 6,5 \\
\hline Posiciones diferentes, sexo oral y masturbación & 4,3 \\
\hline Coito tradicional, juego de roles y otro & 2,2 \\
\hline $\begin{array}{l}\text { Coito tradicional, masajes y relación } \\
\text { con dos mujeres }\end{array}$ & 2,2 \\
\hline Posiciones diferentes, masajes y sexo oral & 2,2 \\
\hline Masajes, sexo oral y sexo anal & 2,2 \\
\hline Coito tradicional, ver pornografía y salir a bailar & 2,2 \\
\hline Servicios compuestos por cuatro actividades & 8,8 \\
\hline $\begin{array}{l}\text { Coito tradicional, posiciones diferentes, } \\
\text { masajes y sexo oral }\end{array}$ & 2,2 \\
\hline $\begin{array}{l}\text { Coito tradicional, masajes, ver pornografía y } \\
\text { otro }\end{array}$ & 2,2 \\
\hline $\begin{array}{l}\text { Coito tradicional, masajes, sexo oral y } \\
\text { masturbación }\end{array}$ & 2,2 \\
\hline $\begin{array}{l}\text { Coito tradicional, sexo oral, conversación y } \\
\text { cenar juntos }\end{array}$ & 2,2 \\
\hline Servicios compuestos por cinco actividades & 2,2 \\
\hline $\begin{array}{l}\text { Coito tradicional, posiciones diferentes, } \\
\text { masajes, sexo oral, y conversación }\end{array}$ & 2,2 \\
\hline Total & 100,0 \\
\hline
\end{tabular}




\section{El mercado}

En el grupo 1, la moda es US\$4 por servicio, en el grupo 2 es de US\$24 y en el grupo 3 asciende a US\$100. Si el cliente desea más tiempo una vez que eyaculó, pasar toda la noche o el fin de semana juntos, debe negociar un pago adicional.

Hay una diferencia importante en las tarifas según el grupo, pero también dentro de cada grupo hay un amplio ámbito entre la tarifa mínima y máxima (Cuadro 14).

Una regresión para relacionar la tarifa a partir de edad y grupo $\left(R^{2}=0,64 p=0,0001\right)$ indicó que la edad no es significativa, apoyando la opinión de las entrevistadas. El grupo de pertenencia es el que define la tarifa (Apéndice 1).

En todos los grupos la entrada a este mercado es por razones económicas (54 de las 58 entrevistadas; una

CUADRO 13

Mujeres entrevistadas según disposición para recibir un pago extra por realizar acciones no deseadas y según uso del preservativo

\begin{tabular}{lccc}
\hline $\begin{array}{c}\text { Uso del } \\
\text { preservativo }\end{array}$ & $\begin{array}{c}\text { Pago extra por acción no } \\
\text { deseada }\end{array}$ & Total \\
& No & Si & \\
\hline No & 2 & 1 & 3 \\
Si & 38 & 6 & 44 \\
Total & 40 & 7 & 47 \\
\hline
\end{tabular}

CUADRO 14

Estadísticos descriptivos de la tarifa (en US\$) por servicio

\begin{tabular}{lccc}
\hline Estadísticos & Grupo 1 & Grupo 2 & Grupo 3 \\
\hline Media & 10,87 & 27,8 & 95,88 \\
Mediana & 6,5 & 28 & 100 \\
Desv. típ. & 12,69 & 5,69 & 35,01 \\
Mínimo & 2 & 20 & 30 \\
Máximo & 50 & 50 & 2000 \\
Ámbito & 48 & 30 & 170 \\
\hline
\end{tabular}

mujer manifestó haber sido obligada por el esposo y dos "por curiosidad").

La mayoría de las mujeres supo que podían trabajar en servicios sexuales y cómo iniciarse, mediante personas conocidas (90\%), rara vez por un anuncio en el periódico y solo en un caso por Internet.

\section{Las decisiones de gasto y ahorro}

Para disponer de una visión de más largo plazo sobre las decisiones económicas de las entrevistadas, se les consultó sobre los ingresos, los gastos y sus prácticas de ahorro.

\section{Ingresos}

Los ingresos mensuales promedio se calcularon a partir de los ingresos semanales reportados por las entrevistadas (calculando 4 semanas por mes). Estos tienen un ámbito muy amplio, con valores extremos de US\$60 y US\$6400. El ingreso mensual promedio en el grupo 1 es de US\$379,50 el del grupo 2 de US\$1100 y el del grupo 3 de US\$2408,89 (uadro 15).

Se pueden obtener ingresos muy altos, pero también muy bajos, lo que le da un carácter financiero riesgoso a este trabajo (Cuadro 15).

La mayoría tiene ingresos considerablemente mayores al salario mínimo de una servidora doméstica o una trabajadora no calificada. Sin embargo, en el grupo 1, de los 16 casos para los que existen datos, en 8 las mujeres tienen un ingreso menor al de una servidora doméstica. En los grupos 2 y 3 , se presenta en cada uno, un caso para el cual el ingreso es menor al salario de una trabajadora no calificada.

CUADRO 15

Estadísticas descriptivas de ingreso mensual (enUS\$)

\begin{tabular}{lcccc}
\hline Estadísticos & Grupo 1 & Grupo 2 & Grupo 3 & Total \\
\hline Mínimo & 60 & 160 & 400 & 60 \\
Máximo & 2560 & 3000 & 6400 & 6400 \\
Ámbito & 2500 & 2840 & 6000 & 6340 \\
Desv. típ. & 599,37 & 751,50 & 1795,82 & 1442,85 \\
Media & 379,5 & 1100 & 2408,89 & 1331,38 \\
Mediana & 190 & 840 & 1800 & 800 \\
\hline
\end{tabular}

\section{Gastos}

Ellas destinan sus ganancias a cubrir las necesidades básicas de la familia: alimentación, ropa, alquiler de vivienda, y pago de servicios públicos. En el grupo 3, además, se incluyen pagos de televisión por cable e internet y los gastos para la educación de los hijos (Cuadro 16).

Una pequeña proporción de los ingresos son dedicados a los cuidados personales, sin embargo, la mayoría, aún en los grupos 2 y 3 donde se reciben altos ingresos, casi no destinan dinero a la recreación ni a la formación profesional. 
Una alta proporción de las trabajadoras de los grupos 2 y 3 indicaron que destinaban parte de sus ingresos al ahorro. En el grupo 1, por el contrario, la mayoría no lo hace (Cuadro 17).

\section{CUADRO 16}

Tipos de gastos en consumo que realizan las entrevistadas con los ingresos percibidos por su actividad (en porcentajes*)

\begin{tabular}{lcccc}
\hline \multicolumn{1}{c}{ Tipos de gastos } & Grupo 1 & Grupo 2 & Grupo 3 & Total \\
\hline $\begin{array}{l}\text { Necesidades básicas } \\
\text { familiares }\end{array}$ & 68,2 & 66,7 & 85,0 & 72,2 \\
Cuidados personales & 13,6 & 26,7 & 5,0 & 4,2 \\
Drogas & 13,6 & - & - & 16,7 \\
Paseos & 4,5 & - & - & 1,4 \\
Estudios & - & 6,7 & - & 2,8 \\
Auto & - & - & 5,0 & 1,4 \\
Recreación & - & - & 5,0 & 1,4 \\
Total & 100,0 & 100,0 & 100,0 & 100,0 \\
\hline
\end{tabular}

*Se calculan los porcentajes respecto al total de menciones.

\section{Dependencia familiar}

Hay una alta dependencia familiar. De la mayoría de estas mujeres dependen los hijos y la madre, pero también deben asumir con la familia extendida en muchos casos. Solo 8 de las 58 entrevistadas no tiene personas que dependan de ellas (Cuadro 18).

\section{Decisiones sobre el uso del dinero}

Las mujeres fueron consultadas sobre el manejo del dinero que ganan en su negocio. Básicamente la administración de los ingresos está en sus propias manos, para todos los grupos, en un $96,4 \%$.

\section{DISCUSIÓN}

Las sociedades patriarcales rechazan por razones ideológicas el que las mujeres puedan elegir el trabajo sexual como su modo de obtener recursos. Sin embargo, la desigualdad económica convierte al trabajo sexual en la única opción que tienen algunas mujeres para obtener recursos con que mantenerse ellas y a sus hijos. En otros casos es una opción deseable debido al monto ganado por hora de trabajo (Schiebinger 1993, Rojas et al. 1994, Leigh 2008). Según el lugar, entre el 0,1 y el $7,4 \%$ de las mujeres adultas del mundo hacen trabajo sexual (Vandepitte et al. 2006) y son por ello víctimas de violencia sicológica (Rojas et al. 1994) basada en la creencia de que son pecadoras,
CUADRO 17

Mujeres entrevistadas por grupos, según realizan o no ahorros (en porcentajes)

\begin{tabular}{lcccc}
\hline $\begin{array}{c}\text { Realizan } \\
\text { ahorros }\end{array}$ & Grupo 1 & Grupo 2 & Grupo 3 & Total \\
\hline $\mathrm{Si}$ & 33,3 & 65,0 & 83,3 & 60,7 \\
No & 66,7 & 35,0 & 16,7 & 39,3 \\
Total & 100 & 100 & 100 & 100,0 \\
\hline
\end{tabular}

CUADRO 18

Dependientes de las mujeres entrevistadas (en porcentajes*)

\begin{tabular}{lcccc}
\hline $\begin{array}{c}\text { Relación de } \\
\text { dependencia }\end{array}$ & Grupo 1 & Grupo 2 & Grupo 3 & Total \\
\hline Hijos-as & $56 \%$ & $47 \%$ & $39 \%$ & $46 \%$ \\
Madre & $15 \%$ & $17 \%$ & $28 \%$ & $20 \%$ \\
Hermanos-as & - & $13 \%$ & $17 \%$ & $11 \%$ \\
Padre & - & $3 \%$ & $6 \%$ & $3 \%$ \\
Abuelos-as & - & $7 \%$ & $3 \%$ & $3 \%$ \\
Otros familiares & $11 \%$ & $3 \%$ & $8 \%$ & $8 \%$ \\
Nadie & $19 \%$ & $10 \%$ & - & $9 \%$ \\
Total de menciones & $100 \%$ & $100 \%$ & $100 \%$ & $100 \%$ \\
\hline
\end{tabular}

* Se calculan los porcentajes respecto al total de menciones

sucias, corruptoras, destructoras y otros epítetos (Herrera 2000). La mitad de las trabajadoras entrevistadas mostró alineación con la creencia de que el trabajo sexual es malo, pero la otra mitad opinó que el trabajo sexual es, para las mujeres, una forma lícita de mantener a sus familias. Además, algunas de ellas indicaron que para los hombres, su trabajo representa un escape que reduce la violencia sexual contra menores de edad y mujeres, en coindencia con los hallazgos de que los escapes sexuales reducen la violencia.

Las creencias populares, mantenidas también por algunos sicólogos y sociólogos de que el trabajo sexual es sinónimo de explotación y hasta esclavitud (e.g. Farley 2004) no se aplican a la población de nuestro estudio, donde las mujeres no tienen jefe y deciden independientemente sobre horario y lugar de trabajo, y sobre cuáles prácticas realizan, rechazando a ciertos clientes si no se llega a un acuerdo.

Tampoco las imágenes de las trabajadoras sexuales comotransmisorasimportantes deenfermedadessexuales, y víctimas de constante y fuerte violencia (Lagarde 2003, 
Farley 2005) calzan con lo informado por nuestras entrevistadas. Con la excepción de las de mayor edad y menor nivel educativo, las trabajadoras contemporáneas de San José se protegen eficazmente con el preservativo. Además, en una proporción importante de los casos, las entrevistadas no reportan casos de violencia.

A diferencia de la sociología y sicología predarwinianas, la sociobiología y la sicología evolutiva consideran al ser humano como parte de la naturaleza, no como un ser superior y aparte del mundo que lo rodea (Foster et al. 2007, Miller et al. 2007, Wilson y Wilson 2007). Nuestros resultados coinciden con las predicciones de los modelos darwinianos (ver Monge-Nájera et al. 2009) en que la mayoría de estas trabajadoras no tienen compañero fijo o esposo que provea suficientes recursos para sus hijos y en que los clientes son hombres con capacidad económica establecida pero todavía en edad reproductiva. Las trabajadoras afirmaron que los clientes son variados, pero con tendencia a estar casados y tener 35-45 años de edad.

Probablemente la mayoría de los clientes no están conscientes de las razones biológicas que los llevan a gastar recursos valiosos (representados en este caso por el dinero pagado, que es convertible en recursos concretos) en actividad sexual (Wilson y Wilson 2007), pero sí de lo que desean, como sexo oral y mujeres con ciertas características físicas (Weitzer 2005). Nuestros resultados calzan con estos deseos, pero no con la idea de que los clientes son hombres con dificultad para establecer relaciones convencionales (Weitzer 2005), ya que la mayoría son casados.

Las mujeres que entrevistamos son emprendedoras en la economía del trabajo sexual, y al violar los preceptos patriarcales de la mujer como objeto al que otros le deciden en qué puede trabajar y en qué no, pagan un alto costo sicológico y social, aunque ya no sufren por ello la cárcel de antaño. La importancia de este sector económico varía según los países (OECD 2002), y en la San José del 2008 es baja, debido a la poca cantidad de mujeres y hombres que participan de él (ver datos en la introducción). Sin embargo, para las mujeres que lo practican, los montos ganados mediante servicios sexuales son la totalidad de su medio de vida, la forma de sacar adelante sus familias y de llevar ellas mismas una vida que no es ni tan fácil ni oscura como la pinta la sociedad en que les correspondió vivir.

\section{RESUMEN}

Aunque el trabajo sexual corresponde a una transacción comercial y es parte de la economía, socialmente se oculta y su flujo de capital no se menciona en los informes económicos. En 2008 entrevistamos a 78 mujeres que lo realizan en la ciudad de San José, capital de Costa Rica. Hubo tres grupos: mujeres que trabajan en la zona aledaña al Mercado Borbón y Mercado Central, con tarifa entre US\$3 y US\$8 por hora y edades de 26 a 81 años; trabajadoras de dos salas de masajes de San Juan de Tibás (US\$18 a US\$72 por hora y 18 a 33 años) y trabajadoras independientes (US\$100 la hora; 19 a 38 años). Las entrevistas se hicieron con base en un cuestionario, se pagó a las entrevistadas su tiempo y los datos se analizaron con el programa estadístico SPSS. Según el grupo, entre la mitad y una mayoría opinó que el trabajo sexual es, para las mujeres, una forma lícita de mantener a sus familias y para los hombres, un escape que reduce la violencia sexual. Las mujeres no tienen jefe y deciden independientemente sobre horario y lugar de trabajo, y sobre cuáles prácticas realizan, rechazando clientes si no hay acuerdo. Pocas han sufrido violencia o enfermedades de transmisión sexual como resultado de su trabajo sexual. La mayoría no tienen compañero fijo o esposo. El cliente preferido es amable, de edad madura y se atiende siempre con la misma mujer. El cliente indeseable es agresivo, tacaño, sucio y/o drogado. Los clientes son principalmente casados y con 35-45 años de edad. Los servicios solicitados son diversos, pero predominan el sexo oral y el coito con la mujer boca arriba. La tarifa es definida por el grupo de pertenencia (mercado, sala o independiente) y no por la edad. Aunque las ganancias varían ampliamente, superan en mucho lo que estas mujeres ganarían en empleos adecuados a su baja escolaridad. Hay poca cultura de ahorro, pero las trabajadoras tienen total decisión sobre en qué gastan sus ganancias, haciéndolo fundamentalmente en el mantenimiento de sus hijos y otros familiares. Debido a las pocas mujeres que realizan trabajo sexual allí, este trabajo representa un porcentaje bajo dentro de la economía de la ciudad de San José.

\section{PALABRAS CLAVE}

Trabajo sexual, economía, mercado, oferta, demanda, precio, ahorro, prostitución.

\section{REFERENCIAS}

Acuña, O., C. Denton, \& F. Naranjo. 1982. El trabajo sexual en San José. Estudio socioeconómico de un problema costarricense. Universidad Nacional, Heredia, Costa Rica.

Becker, G. 1973. A Theory of Marriage. Journal of Political Economy 81: 813-846.

Becker, G. 1976. The Economic Approach to Human Behavior. University of Chicago Press, Chicago, EEUU.

Della G., M., M. Di Tommaso \& S. Strøm. 2005. Who's watching? The market for prostitution services. Centre for Household, Income, Labour, and Demographic Economics. Working Papers $N^{\circ} 16$. Roma, Italia.

Edlund, L. \& E. Korn. 2002. A Theory of Prostitution. Journal of Political Economy 110:181-214.

Farley, M. 2005. Prostitution harms women even if indoors. Violence Against Women 11: 950-964.

Fernández, R. \& J. Rodríguez. 2005. Elementos históricos sobre el trabajo sexual femenino en Costa Rica. El caso del Valle Central Noroccidental. Diálogos: Revista Electrónica de Historia 6:1-43.

Foster, K.R., K. Parkinson \& C.R. Thompson. 2007. What can microbial genetics teach sociobiology? Trends in Genetics 23: 74-80. 
Gertler, P., M. Shah \& S. Bertozzi. 2005. Risky Business: The Market for Unprotected Comercial Sex. Journal of Political Economy 113: 518-550.

Herrera, M. 2000. Detrás del telón...Entre lo imaginario y lo real. Estudio cualitativo sobre mujeres que hacen trabajo sexual. Tesis de Licenciatura. Escuela de Trabajo Social, Universidad de Costa Rica, San José, Costa Rica.

INEC. 2000. IX Censo Nacional de Población. Instituto Nacional de Estadísticas y Censos, San José, Costa Rica.

Lagarde, M. 2003. El feminismo y la mirada entre mujeres. Ponencia en el Seminario Internacional sobre Liderazgo y Dirección para mujeres. "Poder y empoderamiento de las mujeres". Valencia, España.

Leigh, C. 2008. The strange relationship between feminism and sex work. On The Issues Magazine (9 de noviembre 2008). (Disponible en http://www.alternet.org/story/98645/; consultado el 11 de noviembre de 2008).

Marín, H. 2005. La tierra del pecado, entre la quimera y el anhelo: Historia del trabajo sexual en Costa Rica, 1750-2005. Alma Mater, San José, Costa Rica.

Miller, B., J. Tybur \& B. Jordan. 2007. Ovulatory cycle effects on tip earnings by lap dancers: economic evidence for human estrus? Evolution and Human Behavior 28: 375-381.

Monge-Nájera, J., R. Rojas C., R. Morales B. \& I. Ramírez S. 2009. Trabajo sexual femenino en la ciudad de San José, Costa Rica: un enfoque sociobiológico al iniciarse el siglo XXI. Cuadernos de Investigación UNED 1: 27-31.

McKenzie, R. \& G. Tullock. 1978. The New World of Economics: Exploration into the Human Experience. Irwin/McGraw-Hill, Nueva York, EEUU.
OECD. 2002. Measuring the Non-Observed Economy. A Handbook. Organization for Economic Cooperation and Development. OECD Publications Service, París, Francia.

Ortiz, M., A. Zamora, A. Rodríguez, L. Chacón \& A. Gutiérrez. 1998. Soy una mujer de ambiente... Universidad de Costa Rica, San José, Costa Rica.

Posner, R. 1992. Sex and Reason. Harvard University, Cambridge, Massachusetts, EEUU.

Ramírez S., I., J. Monge-Nájera, R. Rojas C. \& R. Morales B. 2009. La escolaridad en trabajadoras sexuales de la ciudad de San José, Costa Rica, al iniciarse el siglo XXI. Cuadernos de Investigación UNED 1: 33-42.

Rojas, A. \& M. Scott. 1994. Relatos de vida y representación del dinero en cinco mujeres prostitutas del sector central de San José. Tesis de Licenciatura, Escuela de Psicología, Universidad de Costa Rica, San José, Costa Rica.

Schiebinger, I. 1993. Nature's body. Gender in the making of modern science. Beakon Press, Boston, Massachussetts, EEUU.

Sisa, L. 2006. He aprendido a vivir bajo el sol. Fundación RAHAB, San José, Costa Rica.

Vandepitte, J., R. Lyerla, G. Dallabetta, F. Crabbé, M. Alary \& A. Buvé. 2006. Estimates of the number of female sex workers in different regions of the world. Sexually Transmitted Infections 82 (Supl. 3): 18-25.

Weitzer, R. 2005. New directions in research on prostitution. Crime, Law \& Social Change 43: 211-235.

Wilson, D.S. \& E.O. Wilson. 2007. Rethinking the theoretical foundation of sociobiology. The Quaterly Review of Biology 82: $327-348$.

Este artículo fue editado por Zaidett Barrientos 


\section{APÉNDICE 1}

Modelo de regresión, tarifa contra grupo de pertenencia Resumen del modelo

\begin{tabular}{cccccc}
\hline Modelo & $\mathbf{R}$ & $\mathbf{R}^{2}$ & $\mathbf{R}^{2}$ corregida & $\begin{array}{c}\text { Error típ. de la } \\
\text { estimación }\end{array}$ & Durbin-Watson \\
\hline 1 &, $814(\mathrm{a})$ &, 662 &, 656 & 12285,291 & 1,840 \\
\hline
\end{tabular}

a Variables predictoras: (Constante), grupo; b Variable dependiente: tarifa por servicio

ANOVA(b)

\begin{tabular}{cccccccc}
\hline Modelo & & Suma de cuadrados & gl & Media cuadrática & F & Sig. \\
\hline 1 & Regresión & 15101455204,871 & 1 & 15101455204,871 & 100,057 &, $000(a)$ \\
& Residual & 7697346681,923 & 51 & 150928366,312 & & \\
& Total & $\mathbf{2 2 7 9 8 8 0 1 8 8 6 , 7 9 3}$ & $\mathbf{5 2}$ & & & \\
\hline
\end{tabular}

a Variables predictoras: (Constante), grupo; b Variable dependiente: tarifa por servicio

\section{Coeficientes(a)}

\begin{tabular}{|c|c|c|c|c|c|c|}
\hline \multirow[t]{2}{*}{ Modelo } & & \multicolumn{2}{|c|}{ Coeficientes no estandarizados } & \multirow{2}{*}{$\begin{array}{c}\begin{array}{c}\text { Coeficientes } \\
\text { estandarizados }\end{array} \\
\text { Beta }\end{array}$} & \multirow{2}{*}{$\begin{array}{l}\mathrm{t} \\
\mathrm{B}\end{array}$} & \multirow{2}{*}{$\begin{array}{c}\text { Sig. } \\
\text { Error típ. }\end{array}$} \\
\hline & & B & Error típ. & & & \\
\hline \multirow[t]{2}{*}{1} & (Constante) & $-20935,927$ & 4636,758 & & $-4,515$ & ,000 \\
\hline & Grupo & 21398,169 & 2139,206 & ,814 & 10,003 & ,000 \\
\hline
\end{tabular}

a Variable dependiente: tarifa por servicio 
